\title{
A practical microfluidic pump enabled by acoustofluidics and 3D printing
}

\author{
Adem Ozcelik $^{1}$ (i) $\cdot$ Zeynep Aslan $^{1}$
}

Received: 24 May 2020 / Accepted: 4 December 2020 / Published online: 4 January 2021

c) Springer-Verlag GmbH Germany, part of Springer Nature 2021

\begin{abstract}
Simple and low-cost solutions are becoming extremely important for the evolving necessities of biomedical applications. Even though, on-chip sample processing and analysis has been rapidly developing for a wide range of screening and diagnostic protocols, efficient and reliable fluid manipulation in microfluidic platforms still require further developments to be considered portable and accessible for low-resource settings. In this work, we present an extremely simple microfluidic pumping device based on three-dimensional (3D) printing and acoustofluidics. The fabrication of the device only requires 3D-printed adaptors, rectangular glass capillaries, epoxy and a piezoelectric transducer. The pumping mechanism relies on the flexibility and complexity of the acoustic streaming patterns generated inside the capillary. Characterization of the device yields controllable and continuous flow rates suitable for on-chip sample processing and analysis. Overall, a maximum flow rate of $\sim 12 \mu \mathrm{L} / \mathrm{min}$ and the control of pumping direction by frequency tuning is achieved. With its versatility and simplicity, this microfluidic pumping device offers a promising solution for portable, affordable and reliable fluid manipulation for on-chip applications.
\end{abstract}

Keywords Microfluidic pump $\cdot$ Acoustofluidics $\cdot$ Acoustic streaming $\cdot$ 3D-printed microfluidics

\section{Introduction}

The ability to drive and control fluid flow in microfluidics is a vital precursor for lab-on-a-chip applications. While conventional fluid manipulation methods, including benchtop syringe pumps and complex pressure-driven setups, provide convenient on-chip experimentation in a well-equipped laboratory, they are not suitable for less than ideal conditions with limited infrastructures that miniaturization and lab-ona-chip platforms actually target. Considering the increasing importance of point-of-care diagnostics and large-scale disease screening (Nasseri et al. 2018; Zhu et al. 2020), precise and practical control of micro- to femtoliters of fluids using simple and cost-effective approaches is even more desired.

Supplementary Information The online version contains supplementary material available at https://doi.org/10.1007/s1040 4-020-02411-w.

Adem Ozcelik

aozcelik@adu.edu.tr

1 Department of Mechanical Engineering, Aydın Adnan Menderes University, Aydın, Turkey
Microfluidics is a thriving research field applied in sample processing (Liu et al. 2017), bioanalysis (Ni et al. 2019), diagnostics (Wang and Lee 2020), and therapeutics (Pollet and den Toonder 2020). There have been numerous studies demonstrating miniaturized microfluidic pumps to facilitate more suitable and applicable on-chip functions for pointof-care applications and low-resource settings (Iverson and Garimella 2008; Gong et al. 2012; Iwai et al. 2014; Li et al. 2019). In this endeavor, various designs and actuation mechanisms have been employed including surface tension (Berthier and Beebe 2007), electric field (Hossan et al. 2018), magnetic field (Malouin Jr et al. 2011; Zhang et al. 2018), optical tweezing (Maruo and Inoue 2007), thermal effects (Tan et al. 2019), periodic volume displacement (Yang et al. 2009; Liu and Lee 2014), and acoustic streaming (Wu et al. 2019b). Surface tension has been explored in various capillary-based microfluidic pumping devices to drive fluid flow in a passive manner (Zimmermann et al. 2007; Chao and Meldrum 2009). A recently presented microfluidic device architecture has been shown to perform capillarybased pumping independent of the surface energy and viscosity of the sample liquid (Guo et al. 2018). They achieved this using the capillary movement of a known working liquid 
located downstream of the microfluidic channel pulling the unknown sample liquid. Another passive microfluidic pumping mechanism has been demonstrated using yarn flow resistance to control the flow rate in a siphon-based microfluidic device (Jeong et al. 2014). They achieved stable flow rates in the range of hundreds of microliters per hour applied in a microfluidic cell incubator. While passive microfluidic pumps are relatively simpler in design and do not usually require complex fabrication and actuation schemes, flow rate metering and control is limited in these systems.

Precise control of flow rates in microfluidics is frequently desired for flexibility and complex sample-handling requirements (Lee et al. 2018). For this reason, various external fields have been labored to achieve fluid pumping and flow rate adjustability by tuning the input field parameters. Due to this pliability and control, this group of microfluidic systems is generally referred as active pumps (Au et al. 2011; Huang et al. 2014). A commonly used mechanism within this group is manipulating fluids by externally applied electric fields also known as electrokinetics (Studer et al. 2002; Li et al. 2019; Yen et al. 2019). Some of the important subcategories of electrokinetic effects are dielectrophoresis, electroosmosis, induced charge electroosmosis, and electrophoresis (Hossan et al. 2018). Electrokinetic methods are well suited for miniaturization and system integration. However, apart from complex fabrication requirements, there are some technical challenges associated with these systems. For instance, one of the main limitation of the these pumps is their dependence on the conductivity of the liquids which may sometimes oblige sample medium modifications to control pumping performance (Vafaie et al. 2016). Another challenge especially encountered in electroosmosis-based micropumps is the generation of bubbles during ionization process which generally affects the device performance adversely (Wang et al. 2009).

Magnetic field has been harnessed for microfluidic pumping in various means including cilia actuation, magnetohydrodynamics, ferrofluidic actuation and membrane actuation (Zhang et al. 2007; Hanasoge et al. 2018; Chen et al. 2020) These approaches generally involve a moving external magnetic field and internal actuation mechanisms which result in more complex device architectures. Similarly, optically driven microfluidic pumps rely on moving parts and fairly complicated laser setups (Leach et al. 2006; Maruo and Inoue 2007; Jung et al. 2014). Thermal gradient-based pumps employ a temperature difference to induce fluid transport in microfluidics (Salari et al. 2019). Flow rate control in such pumps is achieved through adjusting the temperature gradient within the fluid which may not be suitable for biomedical applications due to potential thermal damage to biomaterials (Das et al. 2008). Although peristaltic microfluidic pumps, which have been widely explored for on-chip fluid handling, provide relatively cheaper alternatives that are generally capable of higher flow rates, they have various drawbacks including potential detrimental effects to cells due to pulsatile flows (Li et al. 2013), poor long-time durability due to constant deformations, and limited flow profiles (Stewart 2019).

The field of acoustofluidics, has spawned numerous applications in the last 2 decades from single cell studies to fluid and organism manipulation (Ozcelik et al. 2018; Wu et al. 2019a). The versatility of these systems stems from their non-invasive, low-cost, and label-free attributes (Neild 2016). There are two fundamental forces used in acoustofluidics: acoustic radiation force and acoustic streaming-induced drag force. While acoustic radiation forces mainly arise from a gradient due to scattering, reflection, dampening, interference or absorption of acoustic waves (Bruus 2012; Karlsen et al. 2016), acoustic streaming results from viscous attenuation of acoustic waves inside a fluid medium (Friend and Yeo 2011). Acoustic radiation forces have been conveniently used to manipulate single cells and organisms in various modes (Devendran et al. 2014; Guo et al. 2015; Ayan et al. 2016; Sesen et al. 2017), whereas acoustic streaming has been primarily applied in fluid manipulation in microfluidics (Phan et al. 2016). Different mechanisms have been explored to generate acoustic streaming and fluid pumping including surface acoustic wave-based localized streaming (Wu et al. 2019b), trapped microbubble-based microstreaming (Tovar et al. 2011; Gao et al. 2020), and sharp edge-based acoustic streaming (Huang et al. 2014; Nama et al. 2014; Doinikov et al. 2020). Even though acoustofluidic methods provide reliable, continuous and controllable microfluidic pumping, they require complex and expensive cleanroom fabrication steps which is a limiting factor for low-resource settings and widespread adoptability. Therefore, there is still a need for a simpler and more practical microfluidic pump to address the current challenges.

Herein, we present a new method of acoustofluidic pumping using glass capillary-induced acoustic streaming and 3D-printed architectures. In this work, we address various limitations and challenges of the existing microfluidic pumping platforms by developing an extremely simple fabrication and assembly approach as well as the actuation mechanism. The acoustic streaming profiles generated at various frequencies within the glass capillaries have been studied to achieve flow rate control via tuning the amplitude of the applied acoustic waves. Furthermore, frequency-dependent fluid pumping direction reversal is demonstrated for the first time by employing different eigenmodes of the capillaries. With its simplicity, low-cost and versatility, the presented system carries great potential to be adopted for various on-chip biomedical applications. 


\section{Experimental methods}

The proposed microfluidic pumping device is comprised of a glass capillary tube with a rectangular cross-section, 3D-printed fluidic channels (adaptors), a top connector, and a piezoelectric transducer (Fig. 1a). A fused deposition modeling (FDM) type 3D printer (i3 Mega, Anycubic, Shenzhen, China) is used for 3D printing the plastic parts of the system. The following printing parameters are set in the slicing software; print speed: $50 \mathrm{~mm} / \mathrm{s}$, infill: $100 \%$ with zigzag pattern, cooling: $100 \%$, retraction: enabled, layer height: $0.1 \mathrm{~mm}$, and wall line count: 3 . Standard $1.75-\mathrm{mm}$ diameter polylactic acid (PLA) filament is used with $210^{\circ} \mathrm{C}$ at the print-head and $60{ }^{\circ} \mathrm{C}$ at the print bed. Two adaptors are printed to form a closed-loop complete microfluidic channel geometry. The adaptors are designed to have a top inlet port and a fluidic channel embedded inside that can be connected to two rectangular cross-sectioned capillary tubes (Fig. 1).

For the device assembly, two adaptors are positioned to have their capillary ports facing to each other, and two rectangular glass capillaries (5010, Vitrocom, NJ, USA) with $50 \mathrm{~mm}$ length, $0.1 \mathrm{~mm}$ height, $1 \mathrm{~mm}$ width and $0.07 \mathrm{~mm}$ wall thickness are inserted to each adaptor as shown in Fig. 1b. Afterwards, a 5-min epoxy (E340, Akfix, Istanbul, Turkey) is carefully applied around the edges of the ends of the capillaries to provide liquid-tight sealing. The microfluidic path is completed through the channels embedded inside the adaptors. Subsequently, a piezoelectric transducer (SMPL26W8T07111, Steminc, FL, USA) is bonded on top of one of the capillaries as depicted in Fig. 1. Polyethylene tubes (10793527, Smith's Medical, USA) are inserted into the top inlet and outlet of the adaptors. Finally, the top connector is glued onto the adaptors to provide a robust device assembly. powered by a DC power supply (SPD-3606, GW-Instek, Taiwan). Output signals are measured using a digital oscilloscope (TBS2104, Tektronix, OR, USA). The audio amplifier circuit can amplify sine waves with frequencies around $70 \mathrm{kHz}$ up to $60 \mathrm{~V}_{\mathrm{PP}}$ with minimal distortion of the waveform. The function generator can output $20 \mathrm{~V}_{\mathrm{PP}}$ maximum voltage for waveforms up to $\mathrm{MHz}$ frequencies. As a method of resonance frequency characterization, S11 parameters of the piezoelectric transducers are measured using a portable vector network analyzer (NanoVNA, Huayang, Guangdong, China). Experiments are conducted under an inverted optical microscope (OX.2053-PLPH, Euromex, Arnhem, Netherlands). Still images and videos are recorded using a high-definition CMOS camera (HDUltra, Euromex, Arnhem, Netherlands) and a high-framerate portable camera (Exilim EX-FC100, Casio, Tokyo, Japan). For acoustic streaming and flow characterization, 1 - and 5- $\mu \mathrm{m}$ diameter polystyrene microparticles (Sigma Aldrich, MO, USA) are used in deionized water. Fluidic channels are initially flushed with ethanol to prevent bubble entrapment inside the device. For microfluidic pumping experiments, once filled with the bead solutions, the inlet and the outlet of the device are blocked with solid needles to ensure that the fluid is still in the absence of acoustic excitation. Flow rate characterizations are performed on the bare glass capillary to capture laminar flow for easier bead tracking and analysis.

\section{Results and discussions}

\subsection{Acoustic streaming inside glass capillaries}

Acoustic actuation of the rectangular glass capillary induces various acoustic streaming profiles within the

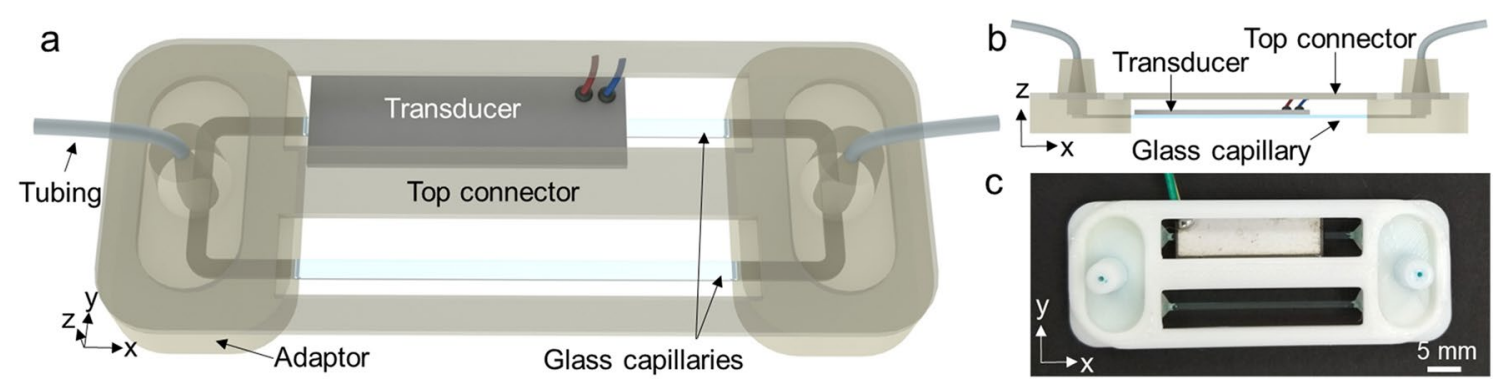

Fig. 1 Demonstration of the acoustofluidic pump assembly and architecture. Schematic depiction of the $\mathbf{a}$ assembled and $\mathbf{b}$ side views are shown. c Photograph of an assembled device

To actuate the acoustofluidic device, a sine wave is applied to the piezoelectric transducer through a function generator (AFG1062, Tektronix, OR, USA) and amplified using a simple TDA7293 100-W audio amplifier circuit fluid domain due to the attenuation of the acoustic energy. As the frequency of the applied acoustic field varies, the streaming patterns inside the capillary change shape, size and location which is visualized using 1- and 5- $\mu \mathrm{m}$ sized 
polystyrene particles (Fig. 2a-f). Frequency sweeping between 55 and $70 \mathrm{kHz}$ and the resulting acoustic streaming patterns in the capillary are shown in Online Resource 1. As illustrated by the red-colored rectangle in Fig. $2 \mathrm{~g}$, these streaming patterns are recorded in the same capillary that the transducer is bonded. For microfluidic fluid pumping applications, directional displacement of the liquid is required. Therefore, streaming patterns generated between 60 and $70 \mathrm{kHz}$ are particularly interesting and potentially useful for this goal because of the fluid streaming directionality observed in these patterns. At higher frequencies such as $187 \mathrm{kHz}$ and $220 \mathrm{kHz}$, rotating vortices are observed in different planes which may be useful for micro-mixing or rotational manipulation applications (Online Resource 2). Characterization of the piezoelectric transducer used in these experiments yields negative peaks in the $\mathrm{S} 11$ parameter around $60 \mathrm{kHz}$ and $200 \mathrm{kHz}$ indicating minimum power reflection which can be correlated to resonance frequency of the transducer (Online Resource 3 Fig. S1) (Wang et al. 2020). The strongest acoustic streaming flows are also observed around these frequencies. It is also worth mentioning that the dimensions of the capillary used in the device influence the generated acoustic streaming patterns. For example, when another capillary with thicker walls is used with the same frequency and amplitude, the acoustic streaming is observed as weaker compared to the presented data. It is expected that as the wall thickness increases, the glass capillary becomes more rigid, and also its resonance behavior changes.

\subsection{Numerical analysis}

In addition to the resonant frequency of the transducer, the eigenmodes of the glass capillaries are also critical in understanding the capillary displacements at different frequencies (Ozcelik et al. 2016). For this purpose, numerical simulations are performed using the COMSOL Multiphysics software. To elucidate the experimentally observed acoustic streaming behavior, first, modal analysis of the glass capillary with the bonded transducers is completed. Since the size and the mass of the transducer is relatively bigger than the glass capillary, eigenmodes of the combined mass of the capillary and the transducer is more realistic and relevant to the experiments. Due to the strong epoxy bonding between the two, we consider the transducer and the capillary as a single body. Some of the different modes are shown in Fig. 3. The same modes with repeating higher orders are also observed within this frequency range. As a comparison, the eigenmodes of the single capillary are shown in Online Resource 3 Fig. S2. For the combined geometry, flexural (Fig. 3a, b, and e), flexural-torsional (Fig. 3c and f), and axial (Fig. 3d) modes are observed from the numerical analysis. From this
Fig. 2 Acoustic streaming profiles inside the rectangular glass capillary are visualized using streak images. At lower frequencies, more specifically at a $61.7 \mathrm{kHz}, \mathbf{b} 62.5 \mathrm{kHz}$, c $63.7 \mathrm{kHz}$ and $\mathbf{d} 67.3 \mathrm{kHz}$, acoustic streaming flows results in more directional fluid displacement within the capillary. At higher frequencies of $\mathbf{e}$ $220 \mathrm{kHz}$ and $\mathbf{f} 187 \mathrm{kHz}$, acoustic streaming-induced flows yield rotating vortices at different planes. In $\mathbf{f}-\mathrm{i}$ and $\mathbf{f}-\mathrm{ii}$, two adjacent vortices are shown side by side because they could not fit in the same frame due their larger spatial distribution in the capillary. Yellow arrows are used to indicate the streaming directions. $\mathbf{g}$ The streaming profiles are recorded from the region of interest (ROI) shown with a red rectangle (color figure online)

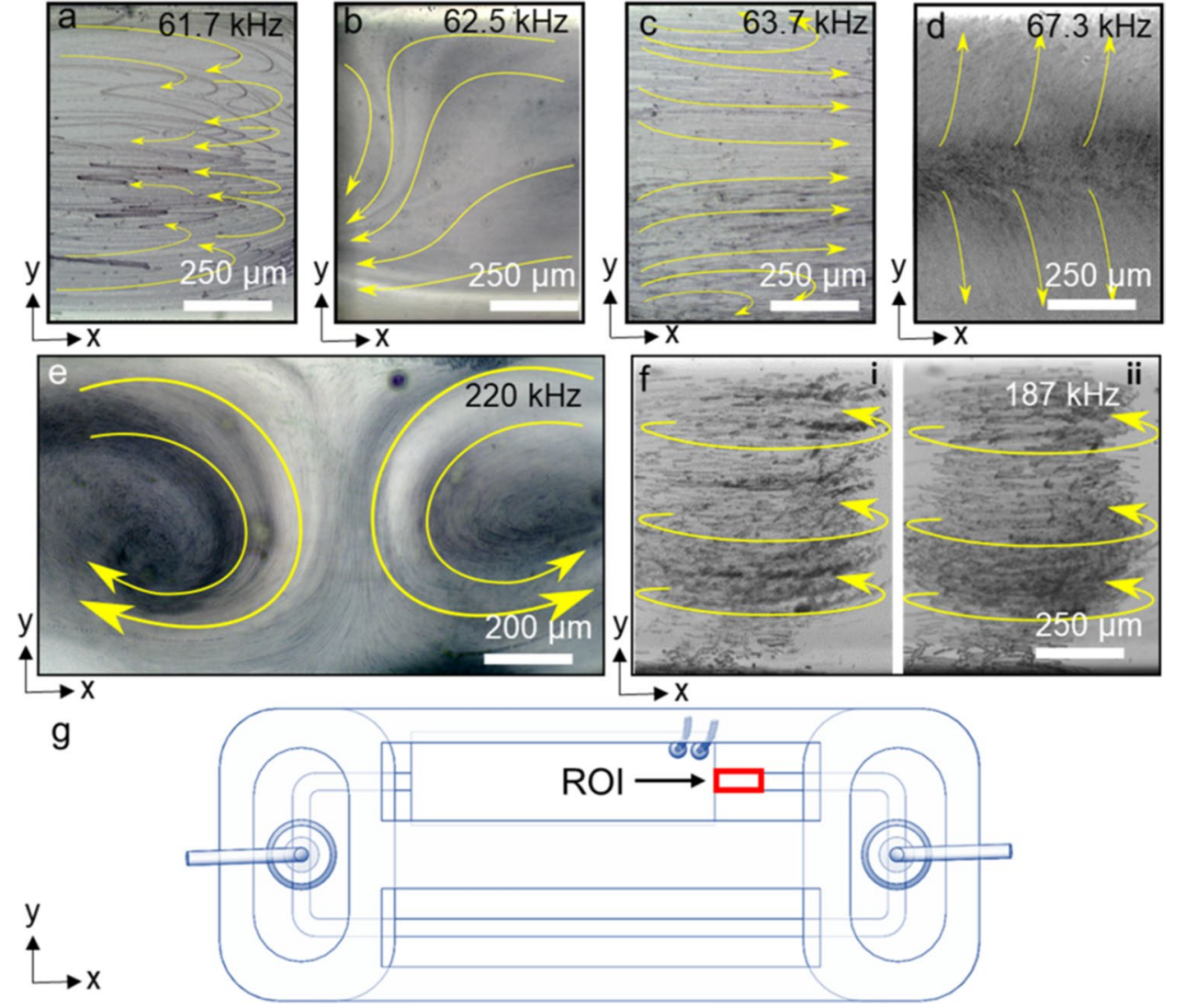


numerical study, displacement of the glass capillary can be visualized, which is believed to be the driving mechanism of the experimentally observed acoustic streaming profiles.

In the next step, the acoustic streaming profiles in the glass capillary are numerically studied. For this, the oscillations occurring at different eigenmodes are considered as the harmonic forcing in the fluid. The response of the fluid can be analyzed by employing the well-known Navier-Stokes equations for a Newtonian, compressible and viscous fluid:

$\frac{\partial \rho}{\partial t}+\nabla \cdot(\rho v)=0$

$\rho \frac{\partial v}{\partial t}+\rho(v \cdot \nabla) v=-\nabla p+\mu \nabla^{2} v+\left(\mu_{b}+\frac{1}{3} \mu\right) \nabla(\nabla \cdot v)$.

Here, $v, \rho, p, \mu$, and $\mu_{\mathrm{b}}$ are velocity of the fluid, mass density, pressure of the fluid, shear dynamic viscosity, and bulk dynamic viscosity, respectively. Equations (1) and (2) express the conservation of the mass and momentum, correspondingly. It is also convenient to write a linear relation between the fluid pressure and the density as $p=c_{\mathrm{o}}^{2} \rho$, where $c_{\mathrm{o}}$ is given as the speed of the sound in the stationary fluid (Nama et al. 2014). Even though the Navier-Stokes equations used with the constitutive relation associating the density and the pressure can fully reveal the fluid response, straightforward numerical simulation of this set of equations with the non-linearity is computationally demanding and also expensive due to the large discrepancy in the typical wavelengths of the acoustic waves compared to the characteristic geometrical dimensions of the system, and time scales of typical oscillation periods compared to the time scale governed by the streaming speed (Nama et al. 2014). Therefore, the set of equations are linearized by a separation order which is a valid conversion in the limit of small amplitude oscillations. That is, the response of the fluid can be analyzed computationally using a perturbation method to expand the flow variables in the Navier-Stokes equations. In this process, the flow variables are divided into the first-order time harmonic and the second-ordered (steady and time-averaged fluid flow; namely acoustic streaming) components. Thus, Nyborg's perturbation method is adapted assuming the following equations for the velocity, density and the pressure of the fluid (Nyborg 1998):

$v=v_{0}+\varepsilon \tilde{v}_{1}+\varepsilon^{2} \tilde{v}_{2}+O\left(\varepsilon^{3}\right)+\cdots$

$p=p_{0}+\varepsilon \tilde{p}_{1}+\varepsilon^{2} \tilde{p}_{2}+O\left(\varepsilon^{3}\right)+\cdots$

$\rho=\rho_{0}+\varepsilon \tilde{\rho}_{1}+\varepsilon^{2} \tilde{\rho}_{2}+O\left(\varepsilon^{3}\right)+\ldots$.

Here, $\varepsilon$ is a dimensionless smallness parameter (Nama et al. 2015). Rewriting Eqs. 1 and 2 with the new forms of the velocity, pressure and density which are given by Eq. 3 results in a first-order equation system:

$\frac{\partial \rho_{1}}{\partial t}+\rho_{0}\left(\nabla \cdot v_{1}\right)=0$
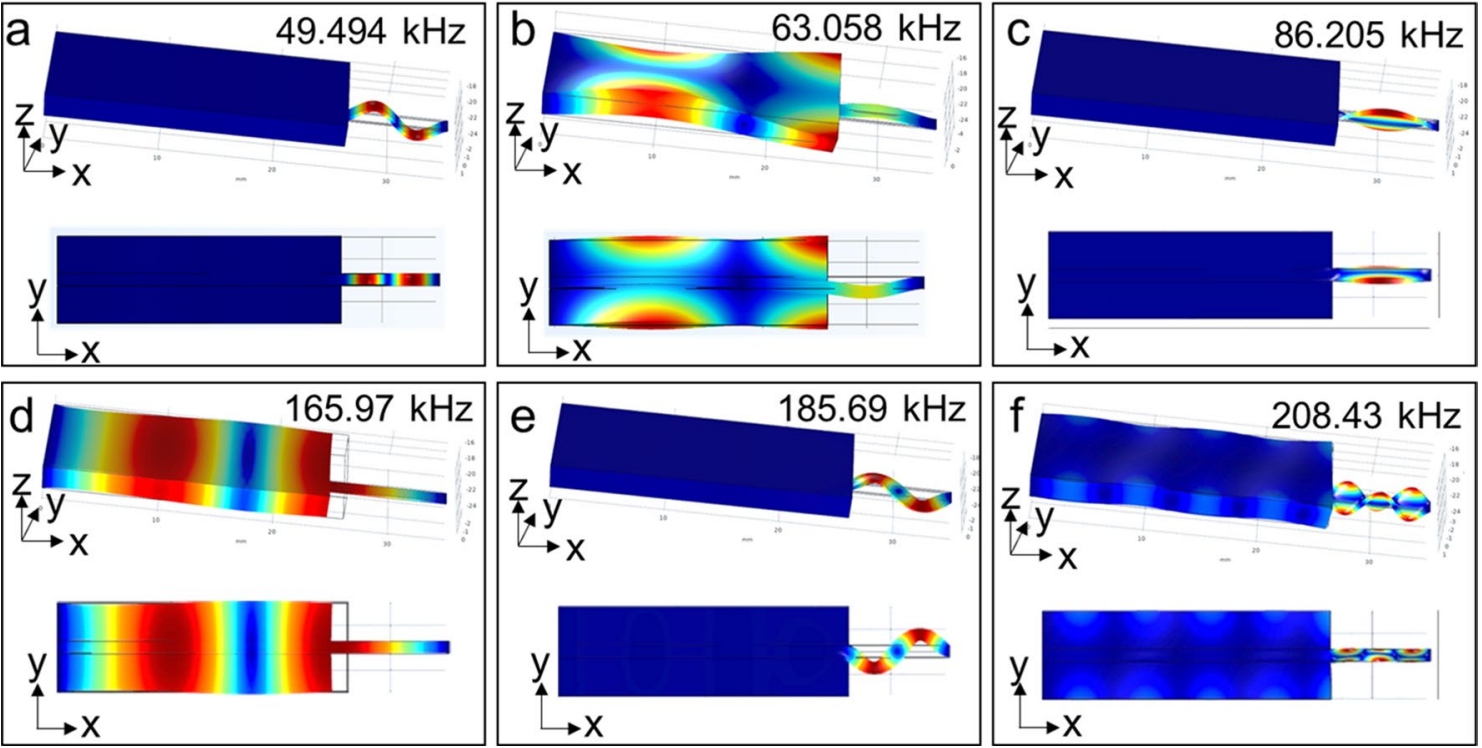

Fig. 3 a-f Eigenmode analysis of the transducer and the glass capillary for the range of frequencies used in the experiments. Due to the geometry of the single body, exposed section of the glass capil- lary generally shows larger displacement for the frequencies used (blue and red color indicates minimum and maximum displacement, respectively) (color figure online) 
$\rho_{0} \frac{\partial v_{1}}{\partial t}=-\nabla p_{1}+\mu \nabla^{2} v_{1}+\left(\mu_{b}+\frac{1}{3} \mu\right) \nabla\left(\nabla \cdot v_{1}\right)$.

After repeating this step one more time, the second-order equations are obtained as the following:

$$
\begin{aligned}
\left\langle\frac{\partial \rho_{2}}{\partial t}\right\rangle+\rho_{0} \nabla \cdot\left\langle v_{2}\right\rangle=-\nabla \cdot\left\langle\rho_{1} v_{1}\right\rangle, & \\
\rho_{0}\left\langle\frac{\partial v_{2}}{\partial t}\right\rangle+\left\langle\rho_{1} \frac{\partial v_{1}}{\partial t}\right\rangle+\rho_{0}\left\langle\left(v_{1} \cdot \nabla\right) v_{1}\right\rangle= & -\nabla p_{2}+\mu \nabla^{2} v_{2}+\left(\mu_{b}\right. \\
& \left.+\frac{1}{3} \mu\right) \nabla\left(\nabla \cdot v_{2}\right) .
\end{aligned}
$$

The first-order system of Eqs. (4 and 5) are solved for time harmonic solutions, and the second-order Eqs. (6 and 7) are solved for steady solutions yielding the time-averaged response of the fluid (acoustic streaming).

Based on the displacement modes in Fig. 3, different oscillation geometries are applied to the fluid in the capillary. For example, when one end of the capillary oscillates along the $y$-axis symmetrically (Fig. 4a), direction verify that an asymmetric forcing appliedal fluid streaming occurs in the open-end capillary. Similarly, if the same regions of the capillary oscillate along the $x$-axis, directional acoustic streaming is generated with slightly recirculating flows close to the top and bottom walls. The oscillation frequency for these cases is chosen as $60 \mathrm{kHz}$ which is relevant to the lowfrequency experimental streaming profiles. If the oscillations are applied only from top or bottom for Fig. $4 a$ and $b$, similar directional streaming simulations are also generated (Online Resource 3 Fig. S3). In the simulations, an asymmetric forcing is applied close to the ends of the capillary to induce directional acoustic streaming (Fig. 4a and b). These types of directional acoustic streaming profiles are observed in the experimental acoustic streaming flows shown in Fig. $2 \mathrm{~b}$ and c. Considering the resonance frequency of the transducer, the mode shown in Fig. $3 \mathrm{~b}$ or a close variation can exert an asymmetric forcing close to the end of the expose capillary. It is important to note that the shape modes shown in Fig. 3 can be drastically changed with a slight shift in the applied frequency. Therefore, in the experimental conditions, we expect shape modes that are distorted to some degree. Nevertheless, simulations in Fig. 4 verify that an asymmetric forcing applied to the capillary can generate directional acoustic streaming which is also experimentally observed at distinct frequencies.

To simulate the recirculating acoustic streaming vortices as shown in Fig. 2e and f, harmonic foresembling the experimentalrcing to the fluid is applied from only one side in the middle section of the capillary as highlighted in Fig. 4c and d. Two acoustic streaming vortices are simulated in $x-y$ plane as experimentally observed in Fig. 2e, when the oscillations are applied from the $100-\mu \mathrm{m}$-high side of the capillary. This mode of oscillations can be applied by the flexural modes occurring in $x-y$ plane as shown in Fig. 3e. If the oscillations are applied from the 1-mm-wide side of the rectangular capillary, two vortices are generated in $x-z$ plane (Fig. 4d) which is experimentally acquired in Fig. $2 \mathrm{f}$ as out-of-plane vortices observed from $x-y$ plane. The oscillation frequency for generating the counter-rotating vortices is chosen as $200 \mathrm{kHz}$ which is around the experimental frequencies applied for Fig. 2e and f. Overall, the simulated counter-rotating vortices in Fig. $4 \mathrm{c}$ and $\mathrm{d}$ are resembling the experimental data in Fig. 2e and f. It should also be noted that when both the top and bottom faces are oscillated in Fig. $4 \mathrm{c}$ and d, four symmetric vortices are resulted (Online Resource 3 Fig. S3). From the comparison of the simulated acoustic streaming (Fig. 4) and experimental streaming profiles (Fig. 2), it can be inferred that the location and the geometry of the harmonic forcing applied to the fluid is

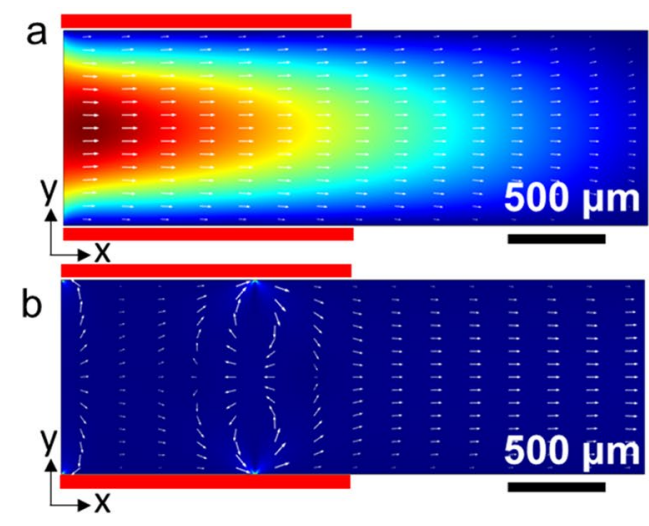

Fig. 4 Numerical simulations of the acoustic streaming patterns in the glass capillaries. Oscillations are applied from the regions highlighted by the red bars. The acoustic streaming velocity field for a vertical (in $y$-axis) and $\mathbf{b}$ horizontal (in $x$-axis) oscillations of the

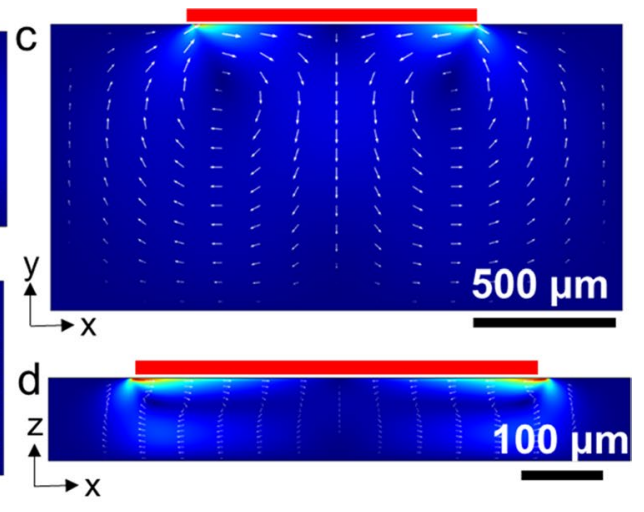

glass capillary. In a different configuration, oscillations are applied from one side of the capillary resulting in two streaming vortices $\mathbf{c}$ on $x-y$ plane and $\mathbf{d}$ on $x-z$ plane 
critical in generating desired fluid actuation behavior inside the glass capillaries.

\subsection{Pumping characterization}

After comparing the acoustic streaming profiles at various frequencies and transducer-binding positions on the glass capillaries, a stable, continuous and controllable fluid pumping behavior is achieved for a particular transducer position at specific excitation frequencies. Directional fluid pumping is characterized in the bare glass capillary that no acoustic excitation is applied. Excitation frequency and oscillation amplitude-dependent fluid pumping behavior is observed when the transducer is bonded on one side of the capillary adjacent to one of the adaptors as shown in Fig. 1. If the transducer is shifted to the center of the capillary (not shown), reproducible directional fluid pumping is not observed in the bare capillary even though acoustic streaming is induced in the capillary that is driven by the transducer. Therefore, it is interpreted that the asymmetrical bonding of the transducer on the rectangular capillary is a required condition in generating controllable and continuous fluid pumping. This can possible be due to the asymmetrical pressure generation inside the capillary at this particular geometry for specific excitation frequencies.

Time series of directional pumping is demonstrated in Fig. 5 for the acoustic parameters of $63.73-\mathrm{kHz}$ excitation frequency and 20 VPP applied voltage (Online Resource 4). At $63.73 \mathrm{kHz}$, maximum fluid pumping performance is achieved in one direction. This frequency is obtained by tuning the frequency from 1 to $300 \mathrm{kHz}$, which also includes the range of the resonant frequencies measured for the transducer, and comparing the generated pumping performances. For this device design and the geometry, another distinct excitation frequency $(61.71 \mathrm{kHz})$ is identified that can generate significant net directional fluid flow in the opposite direction (Online Resource 5). As it can be observed from the Online Resource 5, pumping behavior in both of these frequencies is stable and can be turned on and off by controlling the applied acoustic power. This behavior can be attributed to the variations of the displacement modes by the frequency that in turn can change the harmonic forcing location in the glass capillary.

Fluid pumping performance of the system is characterized at $63.73-\mathrm{kHz}$ and $61.71-\mathrm{kHz}$ driving frequencies resulting in fluid pumping in opposite directions as shown in Fig. 6. For better visualization and tracking, 5- $\mu \mathrm{m}$ diameter polystyrene particles are used in flow rate characterization instead of $1-\mu \mathrm{m}$ particles that are used for acoustic streaming visualization. It is known that as the particle diameter gets larger, the Stokes' drag force also increases which slows down the particles compared to the actual flow rate of the fluid. Therefore, larger than 5- $\mu \mathrm{m}$ particles are not preferred
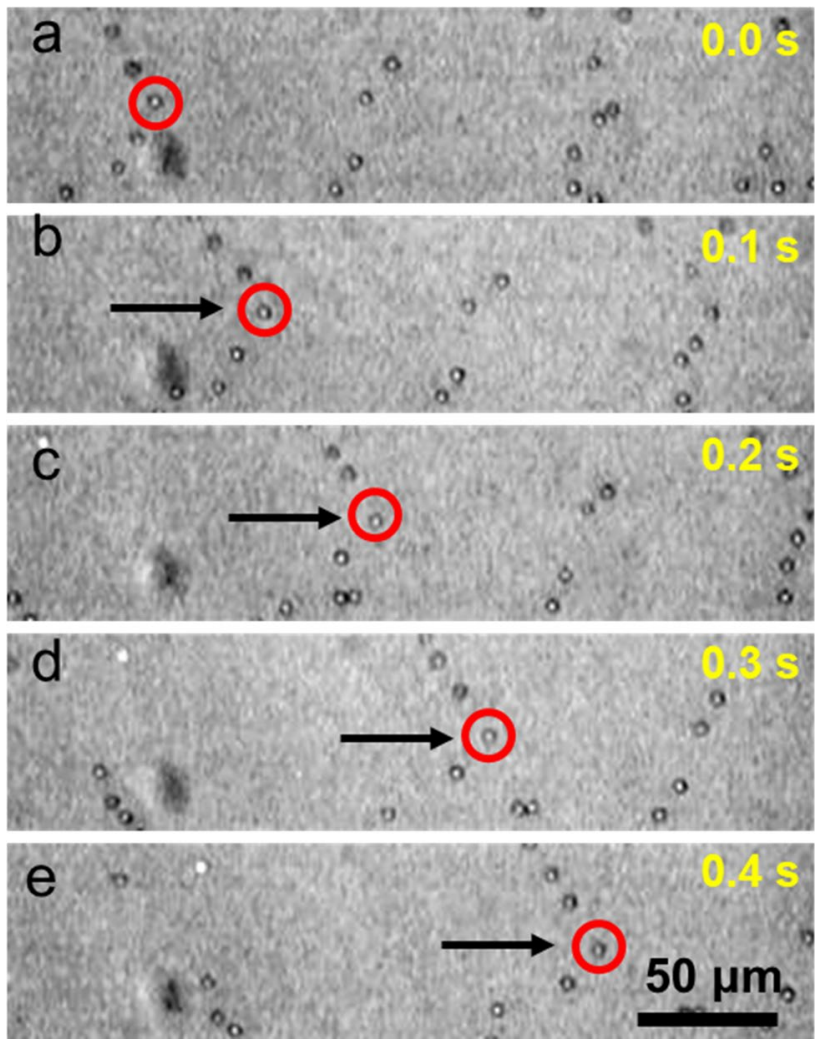

Fig. 5 a-e Time series of directional pumping behavior recorded in the bare glass capillary. The red-colored circle traces a single 5- $\mu \mathrm{m}$ diameter polystyrene particle. Black arrow indicates the flow direction (color figure online)

for flow rate measurements. Theflow rates are measured at increasingly varying applied voltage amplitudes starting from 5 VPP and reaching up to 40 VPP. For the flow rate characterization, particles are tracked for a certain distance under the microscope using the slow motion camera. The travel time for each particle for the measured distance is acquired from the frame count from the start to the end of the distance. Then, the speed of the particle is calculated and multiplied by the inner cross-sectional area of the glass capillary to obtain the volume flow rate. Finally, the flow rate is converted to the more relevant unit of microliters per minute. For each applied voltage, three different experiments are conducted, and minimum 20 particles are tracked from each experiment to calculate the flow rate for one voltage value. While at $63.73-\mathrm{kHz}$ driving frequency, a maximum flow rate of $\sim 12 \mu \mathrm{L} / \mathrm{min}$ is achieved at $40 \mathrm{VPP}$, at $61.71 \mathrm{kHz}$ frequency around $2 \mu \mathrm{L} / \mathrm{min}$ flow rate can be generated at the same driving voltage. Even though the flow rates induced in the opposite direction are much smaller compared to the flow rates occurring at $63.73 \mathrm{kHz}$, the microfluidic pumping system presented here can achieve frequency reversible fluid pumping behavior. In the work of Gao et al., flow reversibility is also demonstrated but the overall maximum flow rate 

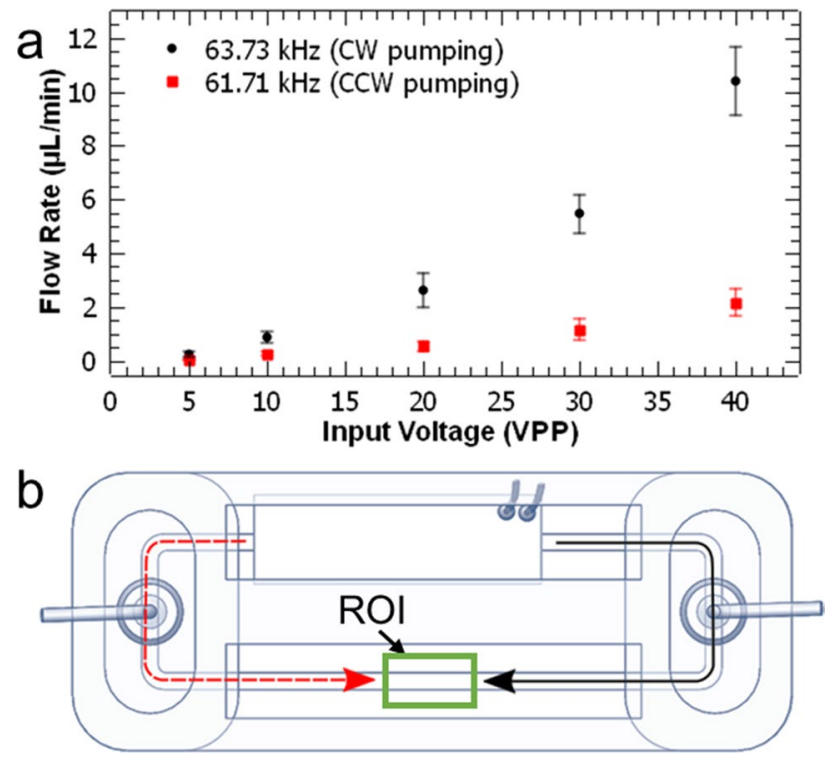

Fig. 6 Characterization of the fluid pumping performance of the proposed device. a Black circles and red squares indicate the flow rates measured for $\sim 63.73 \mathrm{kHz}$ and $\sim 61.71 \mathrm{kHz}$, respectively. b Flow rate characterization is performed from the region of interest (ROI) indicated by the green rectangle. While the black colored arrow shows clockwise pumping, red colored-dashed arrow indicates the counterclockwise pumping behavior. Error bars represents the standard deviation of 60 different calculations from three separate experiments for each point (color figure online)

is reported as $1600 \mathrm{~nL} / \mathrm{min}(1.6 \mu \mathrm{L} / \mathrm{min})$. In the other recent examples of acoustic-based microfluidic pumps, maximum flow rates of $420 \mathrm{~nL} / \mathrm{min}$ (Lin et al. 2019), $41.5 \mathrm{~nL} / \mathrm{min}(\mathrm{Wu}$ et al. 2019b), $8000 \mathrm{~nL} / \mathrm{min}$ (Huang et al. 2014), and $250 \mathrm{~nL} /$ min (Tovar and Lee 2009) are demonstrated. Compared to the existing acoustic-based microfluidic pumps, our device can achieve higher flow rates $(12000 \mathrm{~nL} / \mathrm{min})$ and versatility as well as simplicity in terms of fabrication and assembly. Overall, this system enables adjustable flow rate through controlling the input voltage of the transducer, and flow direction selection by switching the applied frequency.

\section{Conclusions}

Providing rapid and accessible on-chip biomedical analysis including disease diagnostics and testing has been proven to be a crucial step in fighting outbreaks including the COVID19 pandemic that we are experiencing today (Udugama et al. 2020). Therefore, a simple and practical microfluidic pump, which is based on 3D printing and acoustofluidics, is presented that does not require complex and expensive cleanroom fabrication. 3D printing has proven itself as a reliable prototyping platform (Tasoglu and Folch 2018; Kim et al. 2019) even for basic hospital equipment such as ventilators
(Tino et al. 2020). We have also adopted 3D-printing technology to rapidly print and assemble the microfluidic pump using simple glass capillaries and piezoelectric transducers. In addition, we have harnessed the acoustofluidics, which enable non-invasive and label-free manipulation capabilities, in this practical fluid manipulation system.

The characterization of the device yielded precise and active control of flow rates via adjusting the applied voltage to the transducer. A maximum flow rate of $\sim 12 \mu \mathrm{L} /$ min is demonstrated at $40 \mathrm{~V}_{\mathrm{PP}}$ input voltage. Furthermore, control of the flow direction is achieved by exploiting the complex nature of the streaming flows inside the glass capillaries through frequency tuning. Thanks to its simplicity, this microfluidic pump can be integrated to other on-chip platforms to realize truly portable operations. Overall, a versatile platform holding important potential for lab-on-a-chip biomedical applications is demonstrated.

Acknowledgements We greatly thank to Dr. Nitesh Nama, Chuyi Chen, and Dr. Tony Jun Huang for their support and help with the numerical simulations. We also thank Muhammed Kaan Kilinc for his help with 3D printing. This work was supported by The Scientific and Technological Research Council of Turkey (TUBITAK) 3501 grant (Project No. 218M257)

Funding This work was supported by The Scientific and Technological Research Council of Turkey (TUBITAK) 3501 grant (Project No. 218M257).

Availability of data and material Data are available upon reasonable request from the correspond author.

\section{Compliance with ethical standards}

Conflict of interest The authors declare no conflict of interest.

\section{References}

Au AK, Lai H, Utela BR, Folch A (2011) Microvalves and Micropumps for BioMEMS. Micromachines 2:179-220. https://doi. org/10.3390/mi2020179

Ayan B, Ozcelik A, Bachman H et al (2016) Acoustofluidic coating of particles and cells. Lab Chip 16:4366-4372. https://doi. org/10.1039/C6LC00951D

Berthier E, Beebe DJ (2007) Flow rate analysis of a surface tension driven passive micropump. Lab Chip 7:1475. https://doi. org/10.1039/b707637a

Bruus H (2012) Acoustofluidics 7: the acoustic radiation force on small particles. Lab Chip 12:1014. https://doi.org/10.1039/c2lc2 $1068 \mathrm{a}$

Chao S, Meldrum DR (2009) Spontaneous, oscillatory liquid transport in surface tension-confined microfluidics. Lab Chip 9:867. https://doi.org/10.1039/b819887j

Chen Z, Noh S, Prisby RD, Lee JB (2020) An implanted magnetic microfluidic pump for in vivo bone remodeling applications. Micromachines 11:1-10. https://doi.org/10.3390/mi11030300

Das SK, Chung S, Zervantonakis I et al (2008) A microfluidic platform for studying the effects of small temperature gradients in 
an incubator environment. Biomicrofluidics 2:034106. https:// doi.org/10.1063/1.2988313

Devendran C, Gralinski I, Neild A (2014) Separation of particles using acoustic streaming and radiation forces in an open microfluidic channel. Microfluid Nanofluid 17:879-890. https://doi. org/10.1007/s10404-014-1380-4

Doinikov AA, Gerlt MS, Pavlic A, Dual J (2020) Acoustic streaming produced by sharp-edge structures in microfluidic devices. Microfluid Nanofluid 24:32. https://doi.org/10.1007/s 1040 4-020-02335-5

Friend J, Yeo LY (2011) Microscale acoustofluidics: Microfluidics driven via acoustics and ultrasonics. Rev Mod Phys 83:647704. https://doi.org/10.1103/RevModPhys.83.647

Gao Y, Wu M, Lin Y et al (2020) Acoustic bubble-based bidirectional micropump. Microfluid Nanofluid 24:29. https://doi. org/10.1007/s10404-020-02334-6

Gong MM, MacDonald BD, Vu Nguyen T, Sinton D (2012) Handpowered microfluidics: a membrane pump with a patient-tochip syringe interface. Biomicrofluidics 6:044102. https://doi. org/10.1063/1.4762851

Guo F, Li P, French JB et al (2015) Controlling cell-cell interactions using surface acoustic waves. Proc Natl Acad Sci USA 112:43-48. https://doi.org/10.1073/pnas.1422068112

Guo W, Hansson J, van der Wijngaart W (2018) Capillary pumping independent of the liquid surface energy and viscosity. Microsyst Nanoeng 4:2. https://doi.org/10.1038/s4137 8-018-0002-9

Hanasoge S, Hesketh PJ, Alexeev A (2018) Microfluidic pumping using artificial magnetic cilia. Microsyst Nanoeng 4:11. https ://doi.org/10.1038/s41378-018-0010-9

Hossan MR, Dutta D, Islam N, Dutta P (2018) Review: Electric field driven pumping in microfluidic device. Electrophoresis 39:702-731. https://doi.org/10.1002/elps.201700375

Huang P-HH, Nama N, Mao Z et al (2014) A reliable and programmable acoustofluidic pump powered by oscillating sharp-edge structures. Lab Chip 14:4319-4323. https://doi.org/10.1039/ C4LC00806E

Iverson BD, Garimella SV (2008) Recent advances in microscale pumping technologies: a review and evaluation. Microfluid Nanofluid 5:145-174. https://doi.org/10.1007/s1040 4-008-0266-8

Iwai K, Shih KC, Lin X et al (2014) Finger-powered microfluidic systems using multilayer soft lithography and injection molding processes. Lab Chip 14:3790. https://doi.org/10.1039/C4LC0 0500G

Jeong GS, Oh J, Kim SB et al (2014) Siphon-driven microfluidic passive pump with a yarn flow resistance controller. Lab Chip 14:4213-4219. https://doi.org/10.1039/C4LC00510D

Jung JH, Han C, Lee SA et al (2014) Microfluidic-integrated lasercontrolled microactuators with on-chip microscopy imaging functionality. Lab Chip 14:3781. https://doi.org/10.1039/C4LC00790E

Karlsen JT, Augustsson P, Bruus H (2016) Acoustic force density acting on inhomogeneous fluids in acoustic fields. Phys Rev Lett 117:114504. https://doi.org/10.1103/PhysRevLett.117.114504

Kim YT, Bohjanen S, Bhattacharjee N, Folch A (2019) Partitioning of hydrogels in 3D-printed microchannels. Lab Chip 19:3086-3093. https://doi.org/10.1039/C9LC00535H

Leach J, Mushfique H, di Leonardo R et al (2006) An optically driven pump for microfluidics. Lab Chip 6:735. https://doi.org/10.1039/ b601886f

Lee Y-S, Bhattacharjee N, Folch A (2018) 3D-printed Quake-style microvalves and micropumps. Lab Chip 18:1207-1214. https:// doi.org/10.1039/C8LC00001H

Li M, Tan Y, Stenmark KR, Tan W (2013) High pulsatility flow induces acute endothelial inflammation through overpolarizing cells to activate NF-кB. Cardiovasc Eng Technol 4:26-38. https://doi. org/10.1007/s13239-012-0115-5

Li L, Wang X, Pu Q, Liu S (2019) Advancement of electroosmotic pump in microflow analysis: a review. Anal Chim Acta 1060:116. https://doi.org/10.1016/j.aca.2019.02.004

Lin Y, Gao Y, Wu M et al (2019) Acoustofluidic stick-and-play micropump built on foil for single-cell trapping. Lab Chip 19:30453053. https://doi.org/10.1039/c9lc00484j

Liu F, KC P, Zhang G, Zhe J (2017) In situ single cell detection via microfluidic magnetic bead assay. PLoS One 12:e0172697.https ://doi.org/10.1371/journal.pone.0172697

Liu C-H, Lee G-B (2014) A micropump using amplified deformation of resilient membranes through oil hydraulics. Microfluid Nanofluid 17:393-400. https://doi.org/10.1007/s10404-013-1316-4

Malouin BA Jr, Vogel MJ, Olles JD et al (2011) Electromagnetic liquid pistons for capillarity-based pumping. Lab Chip 11:393-397. https ://doi.org/10.1039/c0lc00397b

Maruo S, Inoue H (2007) Optically driven viscous micropump using a rotating microdisk. Appl Phys Lett 91:1-4. https://doi. org/10.1063/1.2768631

Nama N, Huang P-HH, Huang TJ, Costanzo F (2014) Investigation of acoustic streaming patterns around oscillating sharp edges. Lab Chip 14:2824-2836. https://doi.org/10.1039/c4lc00191e

Nama N, Barnkob R, Mao Z et al (2015) Numerical study of acoustophoretic motion of particles in a PDMS microchannel driven by surface acoustic waves. Lab Chip 15:2700-2709. https://doi. org/10.1039/C5LC00231A

Nasseri B, Soleimani N, Rabiee N et al (2018) Point-of-care microfluidic devices for pathogen detection. Biosens Bioelectron 117:112128. https://doi.org/10.1016/j.bios.2018.05.050

Neild A (2016) Motion controlled by sound. Nature 537:493-494. https ://doi.org/10.1038/537493a

Ni L, KC P, Mulvany E, et al (2019) A microfluidic device for noninvasive cell electrical stimulation and extracellular field potential analysis. Biomed Microdev 21:20. https://doi.org/10.1007/s1054 4-019-0364-2

Nyborg WL (1998) Nonlinear acoustics. Academic Press, San Diego

Ozcelik A, Nama N, Huang P-HH et al (2016) Acoustofluidic rotational manipulation of cells and organisms using oscillating solid structures. Small 12:5120-5125. https://doi.org/10.1002/smll.20160 1760

Ozcelik A, Rufo J, Guo F et al (2018) Acoustic tweezers for the life sciences. Nat Methods 15:1021-1028. https://doi.org/10.1038/ s41592-018-0222-9

Pollet AMAO, den Toonder JMJ (2020) Recapitulating the vasculature using organ-on-chip technology. Bioengineering 7:17. https://doi org/10.3390/bioengineering7010017

Salari A, Navi M, Lijnse T, Dalton C (2019) AC Electrothermal effect in microfluidics: a review. Micromachines 10:762. https://doi. org/10.3390/mi10110762

Sesen M, Devendran C, Malikides S et al (2017) Surface acoustic wave enabled pipette on a chip. Lab Chip. https://doi.org/10.1039/ C6LC01318J

Stewart M (2019) Special purpose pumps. In: Surface Production Operations. Elsevier, pp 441-455

Studer V, Pépin A, Chen Y, Ajdari A (2002) Fabrication of microfluidic devices for AC electrokinetic fluid pumping. Microelectron Eng 61-62:915-920. https://doi.org/10.1016/S0167-9317(02)00518-X

Tan Z, Yang M, Ripoll M (2019) Microfluidic pump driven by anisotropic phoresis. Phys Rev Appl 11.

Tasoglu S, Folch A (2018) Editorial for the special issue on 3D printed microfluidic devices. Micromachines 9:609. https://doi. org/10.3390/mi9110609

Tino R, Moore R, Antoline S, et al (2020) COVID-19 and the role of $3 \mathrm{D}$ printing in medicine. 3D Print Med 6:11. https://doi. org/10.1186/s41205-020-00064-7 
Tovar AR, Lee AP (2009) Lateral cavity acoustic transducer. Lab Chip 9:41-43. https://doi.org/10.1039/b812435c

Tovar AR, Patel MV, Lee AP (2011) Lateral air cavities for microfluidic pumping with the use of acoustic energy. Microfluid Nanofluid 10:1269-1278. https://doi.org/10.1007/s10404-010-0758-1

Udugama B, Kadhiresan P, Kozlowski HN et al (2020) Diagnosing COVID-19: the disease and tools for detection. ACS Nano 14:3822-3835. https://doi.org/10.1021/acsnano.0c02624

Vafaie RH, Ghavifekr HB, Van Lintel H et al (2016) Bi-directional ACET micropump for on-chip biological applications. Electrophoresis 37:719-726. https://doi.org/10.1002/elps.201500404

Van PH, Alan T, Neild A (2016) Droplet manipulation using acoustic streaming induced by a vibrating membrane. Anal Chem 88:5696-5703. https://doi.org/10.1021/acs.analchem.5b04481

Wang C-H, Lee G-B (2020) Screening of multiple hemoprotein-specific aptamers and their applications for the binding, quantification, and extraction of hemoproteins in a microfluidic system. Biomicrofluidics 14:024110. https://doi.org/10.1063/1.5141871

Wang X, Cheng C, Wang S, Liu S (2009) Electroosmotic pumps and their applications in microfluidic systems. Microfluid Nanofluid 6:145-162. https://doi.org/10.1007/s10404-008-0399-9

Wang T, Tang Z, Lin $\mathrm{H}$ et al (2020) A low temperature drifting acoustic wave pressure sensor with an integrated vacuum cavity for absolute pressure sensing. Sensors 20:1788. https://doi.org/10.3390/ s20061788

Wu M, Ozcelik A, Rufo J et al (2019a) Acoustofluidic separation of cells and particles. Microsyst Nanoeng 5:32. https://doi. org/10.1038/s41378-019-0064-3

Wu Z, Cai H, Ao Z et al (2019b) A digital acoustofluidic pump powered by localized fluid-substrate interactions. Anal Chem 91:7097-7103. https://doi.org/10.1021/acs.analchem.9b00069
Yang YN, Hsiung SK, Bin LG (2009) A pneumatic micropump incorporated with a normally closed valve capable of generating a high pumping rate and a high back pressure. Microfluid Nanofluid 6:823-833. https://doi.org/10.1007/s10404-008-0356-7

Yen P-W, Lin S-C, Huang Y-C et al (2019) A low-power CMOS microfluidic pump based on travelling-wave electroosmosis for diluted serum pumping. Sci Rep 9:14794. https://doi.org/10.1038/s4159 8-019-51464-7

Zhang C, Xing D, Li Y (2007) Micropumps, microvalves, and micromixers within PCR microfluidic chips: advances and trends. Biotechnol Adv 25:483-514. https://doi.org/10.1016/j.biotechadv .2007.05.003

Zhang S, Wang Y, Lavrijsen R et al (2018) Versatile microfluidic flow generated by moulded magnetic artificial cilia. Sens Actu B Chem 263:614-624. https://doi.org/10.1016/j.snb.2018.01.189

Zhu H, Fohlerová Z, Pekárek J et al (2020) Recent advances in labon-a-chip technologies for viral diagnosis. Biosens Bioelectron 153:112041. https://doi.org/10.1016/j.bios.2020.112041

Zimmermann M, Schmid H, Hunziker P, Delamarche E (2007) Capillary pumps for autonomous capillary systems. Lab Chip 7:119125. https://doi.org/10.1039/b609813d

Publisher's Note Springer Nature remains neutral with regard to jurisdictional claims in published maps and institutional affiliations. 